\title{
Proximal White Onychomycosis in an Immunocompetent Patient: A Case Report
}

\author{
Brian M. Schmidt ${ }^{1}$, Crystal Holmes ${ }^{2}$ \\ ${ }^{1}$ Department of Podiatric Medicine and Surgery, Yale New Haven Hospital/DVA West Haven, New Haven, \\ CT, USA \\ ${ }^{2}$ Department of Internal Medicine, University of Michigan, Ann Arbor, MI, USA \\ Email: bmcsch85@gmail.com
}

Received 29 December 2014; accepted 17 January 2015; published 26 January 2015

Copyright (C) 2015 by authors and Scientific Research Publishing Inc.

This work is licensed under the Creative Commons Attribution International License (CC BY). http://creativecommons.org/licenses/by/4.0/

c) (i) Open Access

\begin{abstract}
Objective: Onychomycosis is a diagnosis of fungal organisms in the nail unit. It frequently occurs in both the immunocompetent and immunocompromised patient. A variant of onychomycosis is proxiaml onychomycosis that occurs on the most proximal aspect of the nail. Proximal onychomycosis is known to be commonly associated with immunocompromised status. Study Design: A case report of a veteran who presented a dermatology clinic with complaint of solitary nail discoloration that was recalcitrant to over-the-counter therapy including topical hydrogen peroxide and foot soaks without improvement. Results and Conclusions: This individual case report demonstrates that pedal proximal onychomycosis can be associated with non-dermatophyte organisms, identified as Fusarium species, in immunocompetent individuals. The finding of pedal proximal onychomycosis caused by a Fusarium non-dermatophytic mold has not been reported. The veteran was successfully cured of the condition with treatment via oral terbinafine for a ninety-day course while monitoring patient's liver function test for any complications.
\end{abstract}

\section{Keywords}

Proximal Onychomycosis, Nail Fungus, Onychcomycosis

\section{Introduction}

Onychomycosis is a diagnosis of fungal infection in the nail caused by dermatophytes, non-dermatophytic filamentous fungi, or yeast species [1]. It occurs frequently in patients that are at a risk of developing fungal infections. These include patients with concomitant fungal infections of the body or foot, people of advanced age, where excessive moisture or warmth occurs, in immunocompromised patients, in people who wear closed and 
occlusive footgear, and in patients with nail deformity or other nail disease [2] [3]. While onychomycosis may present as one disease, it has different presentations and classification of these helps to understand causative organisms.

Onychomycosis can be divided into four groups: distal lateral subungual onychomycosis, proximal white onychomycosis, white superficial onychomycosis, and candidial onychomycosis [4]-[6]. Proximal white onychomycosis is relatively rare compared to the other types of onychomycosis with distal lateral subungual onychomycosis occurring in approximately $90 \%$ of cases [7], while white superficial onychomycosis occurs in $9 \%$ of cases and proximal white onychomycosis and candidial onychomycosis occur less than $1 \%$ of the time [7]. In addition, proximal white onychomycosis is frequently combined with immune compromise status, like HIV positive individuals [8]. In fact, the incidence of proximal white onychomycosis in the immunocompetent population is $0.3 \%$ while proximal white onychomycosis in the HIV-positive population is roughly 15 times greater at $4.2 \%-5.0 \%$ [7] [8].

Below, a case study of toenail proximal white onychomycosis in an immunocompetent individual is demonstrated. This article utilizes the case to demonstrate classic clinical findings and appropriate workup to elucidate if our patient had a systemic illness that was previously underdiagnosed leading to the development of this condition.

\section{Case Study}

A 44-year-old male, a veteran of Desert Storm, presents to the Ann Arbor Veteran Affairs hospital clinic with a two-year history of a white discoloration to his left great toe nail. He denies painful symptoms. He has a remote history of dropping a heavy object on the toe over 10 years ago. His denies previous professional advice but self-treatment included over the counter antifungal creams, hydrogen peroxide application, and soaking the nail without success. His past medical history is significant for post-traumatic stress disorder, GERD, bipolar disorder, migraines and hyperlipidemia. He denies any known drug allergies. He medication list includes lamotrigine, fluoxetine and HCTZ/Trimaterene.

On physical exam the veteran is a well appearing Caucasian male. The neurovascular status is intact in both lower extremities. The proximal half of the left hallux nail plate has white onychomycosis (Image 1). There is no evidence of inflammation or infection to the surrounding skin or proximal nail fold. Otherwise, remainder of distal nail plate is uninvolved. Blood tests, including HIV, hepatitis C, hepatitis B, liver function studies are taken at the initial visit. Fungal cultures of the left hallux nail plate are taken at the time of the initial visit using a small blade to remove shavings from the proximal aspect of the nail. Initial $\mathrm{KOH}$ preparation and screening demonstrated negative results. Upon receipt of tests, all viral assays are negative, LFT's are within normal limits and the preliminary fungal culture confirmed non-dermatophytic mold. This was later confirmed as Fusarium species on the final mycology report. The nail culture did not yield any dermatophytic species.

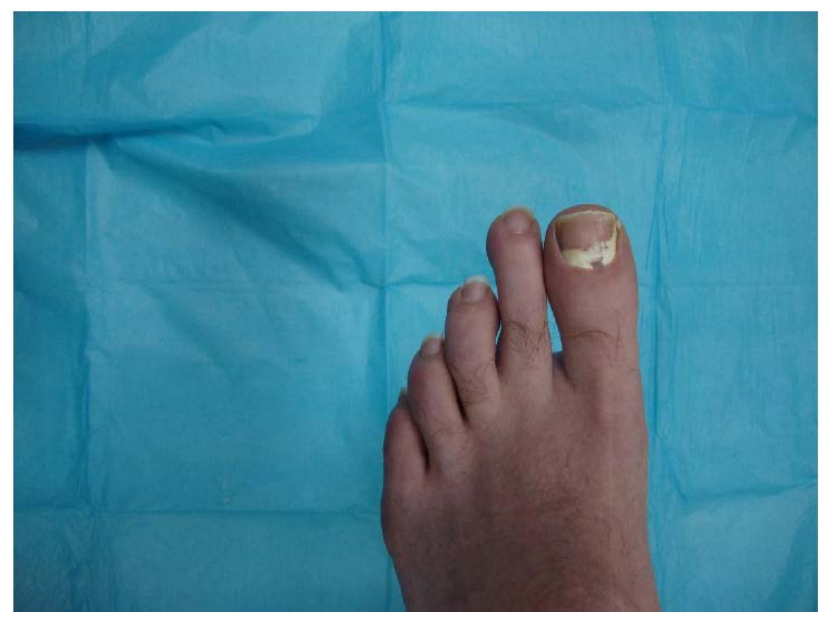

Image 1. Photograph of patient demonstrating white discoloration of the proximal aspect of the nail unit with the distal aspect, including medial and lateral nail folds, uninvolved. 
Patient was started on six weeks of oral terbinafine $(250 \mathrm{mg} /$ day $\times 6$ weeks). After the initial six weeks of therapy, liver function tests were within normal limits and another six weeks prescription of oral terbinafine was written. The patient had complete clinical resolution of symptoms following the second course, ninety days in total, of oral antifungal treatment. The final clinical assessment occurred six months after the initial encounter and three months following completion of the ninety-day course of oral terbinafine.

\section{Discussion}

The prevalence of onychomycosis, all types, is estimated to be around $2 \%-13 \%$ worldwide [9] and between $11 \%-67 \%$, in immunocompromised patients [10]. Specific to proximal white onychomycosis, we see that the majority of cases were reported in patients with AIDS, representing an immunocompromised status. In one study specifically looking at a patient population with AIDS, up to approximately $84 \%$ of patients demonstrated proximal white onychomycosis. However, there has only been a select few case reports of proximal white onychomycosis occurring in immunocompetent patients.

In patients with toenail involvement of proximal white onychomycosis, an immunocompromised status was more common as compared to an immunocompetent patient [11]. In a report of two cases demonstrating immunocompetent patients with proximal white onychomycosis, each patient had only fingernail involvement and with one culture growing Fusarium species and the other T. rubrum with visible hyphae durning staining [12]. Several case reports have showed the occurrence of proximal white onychomycosis in immunocompetent individual but with cultures of Microsporum canis and various Fusarium species being common [13]-[15]. Other articles suggest that $T$. rubrum is the most common cause of proximal white onychomycosis while T. megninii, $T$. schoeleinii, T. tonsurans, T. metagrophytes, and E. floccosum are not uncommon. Our patient only cultured a non-dermatophytic mold as the causative organism in the toenail of this immunocompetent individual [11] [16] [17].

Unlike these case studies, our patient's toenail proximal white onychomycosis culture was positive for nondermatophytic mold growth with Fusarium species as the causative agent. Also, our patient's proximal white onychomycosis occurred on the toenail rather than the fingernail in an immunocompetent individual. The toenail was described as having minimal periungual inflammation but white proximal nail discoloration and leukonychia. An article by Tosti et al. [18], discovered that 59 of their 1548, or roughly 3.8\% patients, had onychomycosis from a causative organism of mold. Specifically, in sixteen cases of proximal onychomycosis, the causative agents were either Scopulariopsis brevicaulis $(\mathrm{n}=10)$ and Aspergillus species $(\mathrm{n}=6)$ [18], neither of which were seen in our case. Hilmioqiu et al. [19] showed that molds were detected in 33 (9\%) with a majority of the cases involving mold occurring in the toenails (67\%). Similarly in their review of 15,000 patients in Canada, Gupta et al. [20] demonstrated that the organisms causing toenail onychomycosis were approximately $7.8 \%$ non-dermatophyte molds.

As mentioned, the patient presented a case of toenail proximal white onychomycosis. He was neither AIDS nor HIV, HBV, HCV positive, and did not demonstrate diabetes mellitus or any other immune suppressed disease state. As proximal white onychomycosis occurs in only $0.3 \%$ of the immunocompetent population [7] [8], our patient is the first reported case of proximal white onychomycosis from non-dermatophytic mold Fusarium species affecting the toenail in an immunocompetent individual. The individual was successfully treated with a ninety-day course of oral terbinafine ( $250 \mathrm{mg}$ /day PO $\times 90$ days) while monitoring liver function initially and at a six-week time interval during the course.

\section{References}

[1] Ahn, K.J. (1994) A Case of White Superficial Onychomycosis. Korean Journal of Dermatology, 32, 931-933.

[2] Habif, T.P., Ed. (2010) Clinical Dermatology. 5th Edition, Saunders Elsevier, Philadelphia, 491-523.

[3] Hay, R.J. (2009) Dermatophytosis and Other Superficial Mycoses. In: Mandell, G.L., Bennett, J.E. and Dolin, R., Eds., Mandell, Douglas, and Bennett's Principles and Practice of Infectious Diseases, 7th Edition, Churchill Livingstone Elsevier, Philadelphia, Chapter 267.

[4] Phillips, P. (1996) New Drugs for the Nail Fungus Prevalent in Elderly. JAMA, 276, 12-13. http://dx.doi.org/10.1001/jama.1996.03540010014007

[5] Elewski, B.E. and Hay, R.L. (1996) Update on the Management of Onychomycosis: Highlights of the Third Annual International Summit on Cutaneous Antifungal Therapy. Clinical Infectious Diseases, 23, 305-313. 
[6] Zaias, N., Glick, B. and Rebell, G. (1996) Diagnosing and Treating Onychomycosis. The Journal of Family Practice, 42, 513-518.

[7] Gupta, A.K., Taborda, P., Taborda, V., Gilmour, J., Rachlis, A., Salit, I., et al. (2000) Epidemiology and Prevalence of Onychomycosis in HIV-Positive Individuals. International Journal of Dermatology, 39, 746-753. http://dx.doi.org/10.1046/j.1365-4362.2000.00012.x

[8] Tlacuilo-Parra, A., Guevara-Gutierrez, E., Mayorga, J., Garcia-De La Torre, I. and Salazar-Paramo, M. (2003) Onychomycosis in Systemic Lupus Erythematosus: A Case Control Study. The Journal of Rheumatology, 30, 1491-1494.

[9] Schlefman, B.S. (1999) Onychomycosis: A Compendium of Facts and a Clinical Experience. The Journal of Foot \& Ankle Surgery, 38, 290-302. http://dx.doi.org/10.1016/S1067-2516(99)80072-6

[10] Odom, R.B. (1996) New Therapies for Onychomycosis. Journal of the American Academy of Dermatology, 35, S26S30. http://dx.doi.org/10.1016/S0190-9622(96)90067-5

[11] Elewski, B.E. (1993) Clinical Pearl: Proximal White Subungual Onychomycosis in AIDS. Journal of the American Academy of Dermatology, 29, 631-632. http://dx.doi.org/10.1016/S0190-9622(08)81867-1

[12] Weinberg, J.M., Koestenblatt, E.K., Don, P.C., White, S.M., Stein, M.N. and Bamji, M. (1999) Proximal White Subungual Onychomycosis in the Immunocompetent Patient: Report of Two Cases and Review of the Literature. Acta Dermato-Venereologica, 79, 81-95.

[13] Piraccini, B.M., Morelli, R., Stinchi, C. and Tosti, A. (1996) Proximal Subungual Onychomycosis Due to Microsporum canis. British Journal of Dermatology, 134, 175-177. http://dx.doi.org/10.1111/j.1365-2133.1996.tb07863.x

[14] Baran, R., Tosti, A. and Piraccini, B.M. (1997) Uncommon Clinical Patterns of Fusarium Nail Infection: Report of Three Cases. British Journal of Dermatology, 136, 424-427. http://dx.doi.org/10.1111/j.1365-2133.1997.tb14959.x

[15] Dordrain-Bigot, M.L., Baran, R., Baixench, M.T. and Bazex, J. (1996) Fusarium Onychomycosis. Annales De Dermatologie et De Venereologie, 123, 191-193.

[16] Silver-Lizama, E. and Logemann, H. (1996) Proximal White Subungual Onychomycosis in AIDS. International Journal of Dermatology, 35, 290-291. http://dx.doi.org/10.1111/j.1365-4362.1996.tb03006.x

[17] Noppakun, N.M. and Head, E.E. (1986) Proximal White Subungual Onychomycosis in a Patient with Acquired Immune Deficiency Syndrome. International Journal of Dermatology, 25, 586-587. http://dx.doi.org/10.1111/j.1365-4362.1986.tb04705.x

[18] Tosti, A., Piracinni, B.M. and Lorenzi, S. (2000) Onychomycosis Caused by Nondermatophytic Molds: Clinical Features and Response to Treatment of 59 Cases. Journal of the American Academy of Dermatology, 42, 217-224. http://dx.doi.org/10.1016/S0190-9622(00)90129-4

[19] Hilmioğlu-Polat, S., Metin, D.Y., Inci, R., Dereli, T., Kilinç, I. and Tümbay, E. (2005) Non-Dermatophytic Molds as Agents of Onychomycosis in Izmir, Turkey-A Prospective Study. Mycopathologia, 160, 125-128. http://dx.doi.org/10.1007/s11046-005-6872-z

[20] Gupta, A.K., Jain, H.C., Lynde, C.W., Macdonald, P., Cooper, E.A. and Summerbell, R.C. (2000) Prevalence and Epidemiology of Onychomycosis in Patients Visiting Physicians' Offices: A Multicenter Canadian Survey of 15,000 Patients. Journal of the American Academy of Dermatology, 43, 244-248. http://dx.doi.org/10.1067/mjd.2000.104794 
Scientific Research Publishing (SCIRP) is one of the largest Open Access journal publishers. It is currently publishing more than 200 open access, online, peer-reviewed journals covering a wide range of academic disciplines. SCIRP serves the worldwide academic communities and contributes to the progress and application of science with its publication.

Other selected journals from SCIRP are listed as below. Submit your manuscript to us via either submit@scirp.org or Online Submission Portal.
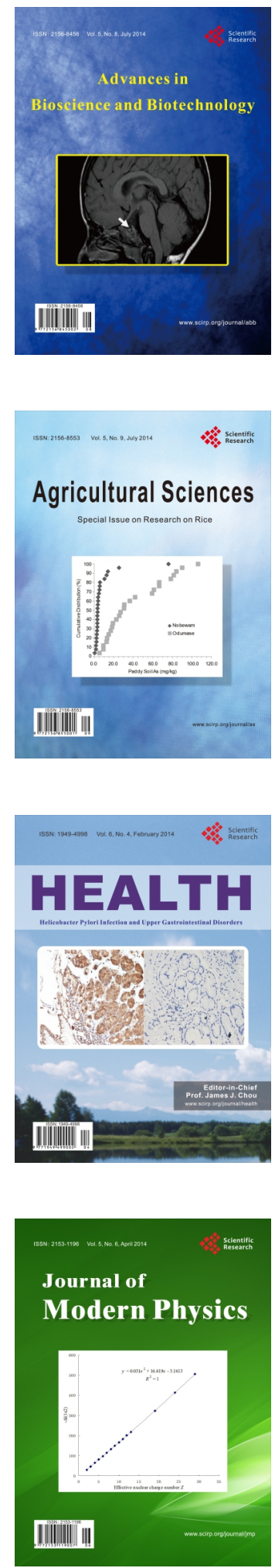
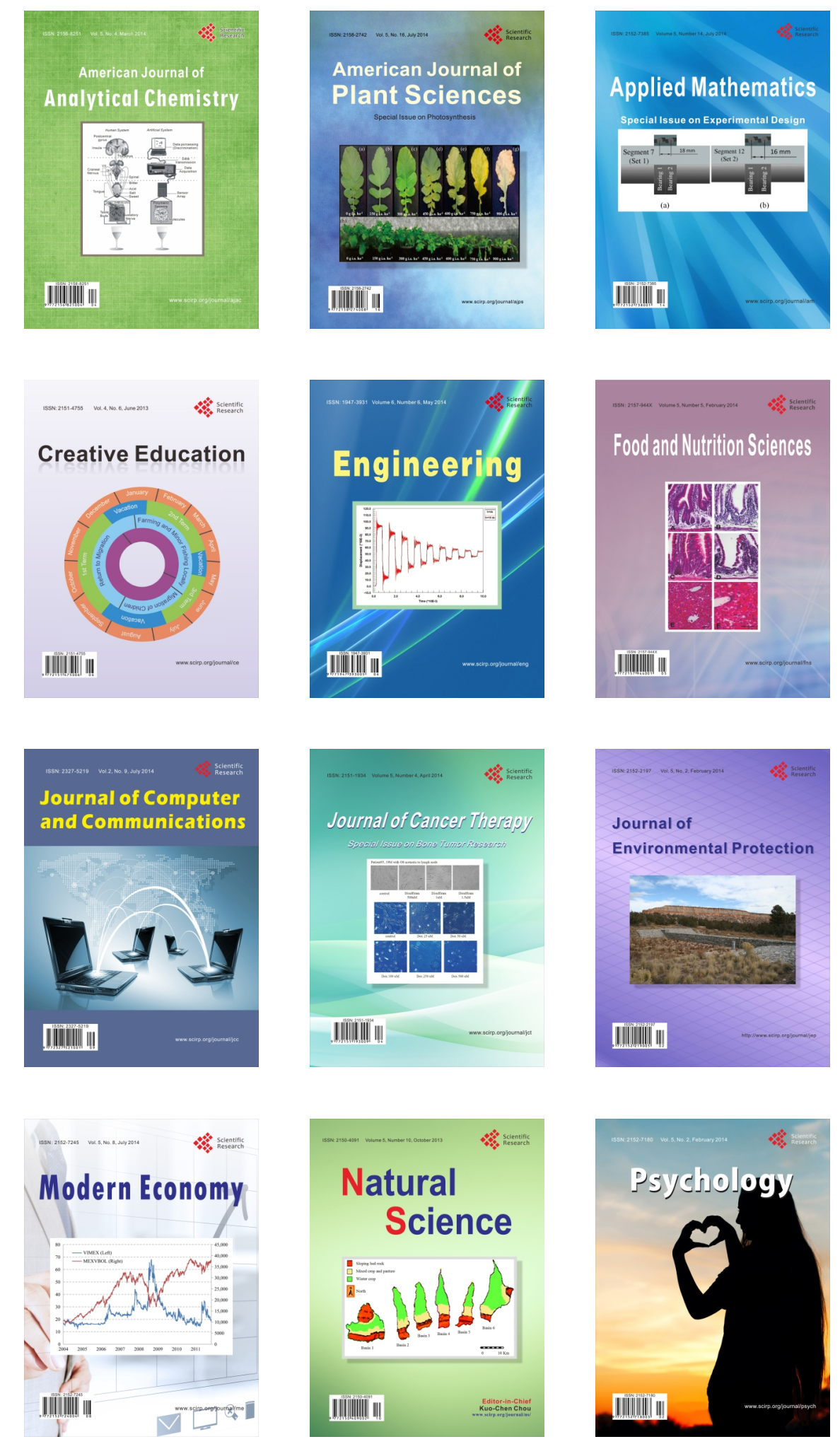